\title{
Interactive Exploration of Geospatial Network Visualization
}

\section{Till Nagel}

Interaction Design Lab, University

of Applied Sciences Potsdam

14469 Potsdam, Germany

nagel@fh-potsdam.de

\section{Erik Duval}

Dept. Computer Science, KU Leuven

3001 Heverlee, Belgium

erik.duval@cs.kuleuven.be

\section{Andrew Vande Moere}

Dept. Architecture, Urbanism

and Planning, KU Leuven

3001 Heverlee, Belgium

andrew.vandemoere@asro.kuleuven.be

Permission to make digital or hard copies of all or part of this work for personal or classroom use is granted without fee provided that copies are not made or distributed for profit or commercial advantage and that copies bear this notice and the full citation on the first page. To copy otherwise, or republish, to post on servers or to redistribute to lists, requires prior specific permission and/or a fee.

CHI'12, May 5-10, 2012, Austin, Texas, USA.

Copyright 2012 ACM 978-1-4503-1016-1/12/05...\$10.00.

\begin{abstract}
This paper presents a tabletop visualization of relations between geo-positioned locations. We developed an interactive visualization, which enables users to visually explore a geospatial network of actors. The multitouch tabletop, and the large size of the interactive surface invite users to explore the visualization in semi-public spaces.

For a case study on scientific collaborations between institutions, we applied and improved several existing techniques for a walk-up-and-use system aimed at scientists for a social setting at a conference. We describe our iterative design approach, our two implemented prototypes, and the lessons learnt from their creation. We conducted user evaluation studies at the two on-location demonstrations, which provide evidence of the prototype usability and usefulness, and its support for understanding the distribution and connectivity in a geospatial network.
\end{abstract}

\section{Author Keywords}

geo-visualization; tabletop interfaces; human computer interaction; exploration; interactive maps

\section{ACM Classification Keywords}

H.5.2 [Information Interfaces And Presentation]: User Interfaces - Interaction styles; 


\section{Introduction}

Physical gatherings at conferences are an important form of scholarly communication, in order to ease socialization, and getting involved in the debate on newest research [35]. One of the main effects for attendees is to be connected to a network of researchers [36], and to establish personal contacts [25].

Our large tabletop display acts as social space for people to gather in a conference setting, be it as bystanders or as active users, and invites them to engage in conversations on location. We opted to visualize research collaboration based on co-authorship data, in order to act as a catalyst for starting casual and opportunistic discussions on the community, and facilitate understanding and reflecting on one's own and others' research network.

There has been a vast amount of research in the areas of bibliometry to extract and specify the metrics of scientific publication and citation networks. Several approaches to visualize these networks have been reported on (e.g. [33], [16]). In contrast, the objective of our case study is not to investigate individual authors and their personal co-authorship networks, but rather to enable analyzing the collaboration network of their affiliations. More specifically, our aim is to direct attention to the spatial relations to enable users to visually explore their scientific neighborhood in order to investigate the characteristics of their network within an existing social setting of a conference.

Geovisualization provides tools for visual exploration of geospatial data, and supports "visually-enabled information retrieval" [23]. The display of geo- positioned objects on a map helps viewers seeing realworld clusters in the visualized data set. The spatial distribution of the data, as well as the visual encoding of data values allows users to detect density patterns. Connections between nodes are based on the semantic relation between objects. Our prototype visualizes bidirectional relations based on a shared data value.

Instead of creating a system with all the possibilities complex geographic information systems (GIS) offer, we intended to develop a reduced and engaging geovisualization with a narrow focus for a very specific use case. In particular, the design of the visualization technique was specifically motivated to facilitate the understanding of geospatial data for a wide audience, including people without expertise in GIS.

Our interactive tabletop visualization is intended to be a walk-up-and-use system. In our case study on research collaboration, the targeted audience mainly consist of attendees at scientific conferences. One of our main design goals for visualization and multitouch interaction was to facilitate first-time users to use the system without training. For this purpose, we created two prototypes aiming to create an easy-to-use interactive geospatial network visualization.

Although it has received relatively little attention in literature, interaction in visualization is one of the main determinants for the quality of a user's dialogue with the data, which ultimately facilitates the understanding and insight into this data. Therefore, the design of the interaction capabilities of our prototypes was based on a set of design guidelines for fluidity in visualization, including: the use of animated transitions, the immediate feedback, the minimalization of indirection, 


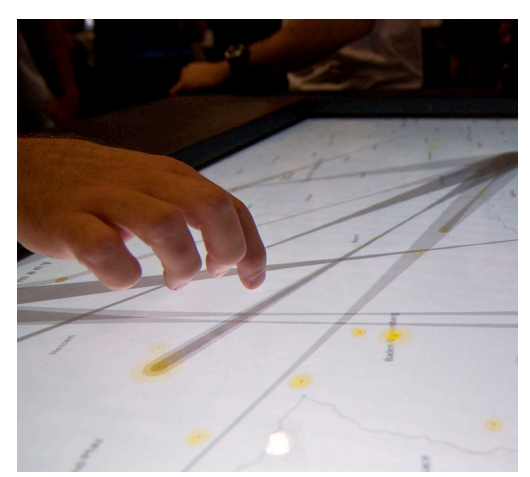

Fig. 1: A pinch gesture to zoom the map. the integration of user interface elements, and neverending exploration possibilities [11].

\section{Related Work}

In scientrometrics, studies have demonstrated the growth of international collaboration in science by using co-authorships (e.g. [7], [13], [22]). One of the objectives is to discover what kind of, how many, and between whom scientific collaboration exists. Studies have shown that geographic proximity is important and does positively influence the intensity and frequency of scientific collaboration [18]. In Börner's Atlas of Science, she states that citation frequency between affiliations decrease the more distant they are [5]. She claims that spatial proximity facilitate building and maintaining personal relationships, which seems to be even more relevant for co-authorship than co-citation networks.

Geo-spatial relations in citation or co-authorship networks have been analyzed by means of visualization (e.g. [2], [30]). As data can be structured in combination with their geo-location in a natural and intuitive way [6] we deem the approach of using geovisualization as very promising. In one study, the top 100 cited researchers have been analyzed by mapping their locations with circles proportional to the number of cited scientists, in order to compare different places [2]

In recent years, several approaches to visually represent collaboration between institutions have been studied. One project used a geographic map highlighting the research collaborations of the Chinese Academy of Sciences with locations in China and countries around the world [9]. Flow lines representing these relations are displayed on a large choropleth map, which is accompanied by six smaller ones, showing collaboration in province-level administrative divisions. In another project a map of scientific collaboration was designed with data from scientific journal aggregator Elsevier Scopus [3]. The map traces the lines of collaboration between cities, which results in a dense and beautiful world network.

We aim to extend some of the insights these static visualizations support, by allowing further examination of the network by selecting geographical areas, and filtering the dataset for exploration of personally relevant sub-networks.

\section{Interactive Prototypes}

The two tabletop prototypes share many characteristics. Both show a world map on the interactive display, with all institutions shown as markers at their geo-locations. The map can be navigated freely, while place markers can be selected to get background information on publication output as well as their relations to other institutions.

In the following paragraphs, we describe the data used, the general visualization and interaction, the geographic maps used, and the tabletop. In sections below, we describe the features of the two prototypes, and explain the pros and cons of the approaches.

\section{Data Acquisition}

The inter-institutional relationships are based on coauthor data, as co-authorship reflects research collaboration between affiliations and countries, adequately [13]. 
We applied our geospatial network visualization in two case studies on collaboration between research institutions. These case studies are based on coauthorship data as stored within two distinct data sets:

For the demonstration of the first prototype, we used the European Conference on Technology Enhanced Learning (EC-TEL) publication data from the years 2006-2010, to show the connectivity in the scientific TEL community. While the dataset of this younger conference was small, it allowed us to demonstrate a prototype visualizing relevant data to conference attendees. We scraped the publication data from the website of Springer, the proceedings publisher. For the demonstration of the second prototype at the ACM Conference on Hypertext and Hypermedia (Hypertext) 2011, publication data from the ACM digital library of the years 1989-2011 were used.

In both cases, we needed to harvest data on coauthorship using our own algorithm, as existing aggregation services such as Bibsonomy [17], citeUlike [8], or Mendeley [26] do not provide affiliation data. Thus, we used Web-Harvest [38] to collect author affiliations and their postal addresses directly from the publisher of the conference proceedings. As the information originally is provided by the authors, using various languages, formats, and accuracy levels, we needed to apply different aggregation and unification heuristics, trying to reduce unintentional duplicates or other skewed data entries.

\section{Cleaning the Data}

First, the affiliation line is split up into the affiliation name and the address, to allow a better unification of affiliations, and to display a shorter and more readable name in the visualization. The simplistic, language agnostic approach was to concatenate all text segments up to and including the last segment containing one of a set of specific keywords, selected for high probability of matching institutional name segments.

Secondly, the affiliations were unified, based on the similarity of the name. The affiliation text for the same institution may be in English or in the original language, may include abbreviations or be written out, and the order of organizational subparts may change between different papers. Thus, in order to identify affiliation duplicates the similarity algorithm needed to have the following characteristics: (1) Strings with small differences should be recognized as being similar. (2) It should be robust to changes in word order. (3) It should be language independent. We used the algorithm developed by White [39], which calculates how many adjacent character pairs are contained in both strings. We considered all pairs of institution names more similar than the threshold of 0.7 to be matches, and all pairs less similar to be non-matches. The chosen threshold value was based on our manual review of the dataset, to be fitting for our specific set of institution names.

Finally, the institution's address was geo-coded. Besides geo-locations, the corresponding countries were stored to allow comparing country-level statistics, later on.

\section{Visualization \& Interaction}

Users are able to select the region they are interested in by panning and zooming the map through slide and pinch finger gestures (see Fig. 1 in margin column). Even though more complex map manipulations are 
possible, we chose this simple interaction approach, in order to enable the user to concentrate on the map, with less efforts.

Affiliations are represented by circular markers at their geo-location. The size of a circle indicates the overall amount of papers written by authors from that institution. In the lower left corner a legend explaining the size of the circles is shown (see Fig. 3 ).

Users can select a country by tapping on the background map within its political borders. That country gets selected, and additional information is shown in a data widget in the lower right corner. The number of papers, authors, and institutions over the years are displayed as bar diagrams (Fig. 3). As long as the user has not tapped anywhere, the widget displays a semitransparent message to communicate this interaction pattern. When two countries are selected the prototype displays the diagrams besides each other, allowing the user to compare them. A second country is selected only if users tap on a different country.

By tapping on a circle, the name of its institution is displayed atop, and relations to other institutions are shown. Relations between institutions are visualized by connecting lines between the two markers. We adhered to the schema most visualizations of social networks use, in that actors are represented as dots, and relations among them as lines [12]. The visual lines connect two institutions transparently, to not obstruct the underlying map or markers.

\section{Geographical map}

The cartographic information shown in the background map originates from OpenStreetMap [15], while

MapBox [24] provides the image tiles. Thus, we were able to customize the map according to interface design requirements, and embed it as interactive maps fast and effortlessly without the technical setup of a complete map server stack.

\section{Tabletop display}

The geo-visualization is shown on an interactive tabletop with multitouch capabilities. With the large interactive surface, the user not only views and manipulates data on a single user system, but operates in a collaboratively created and used information space.

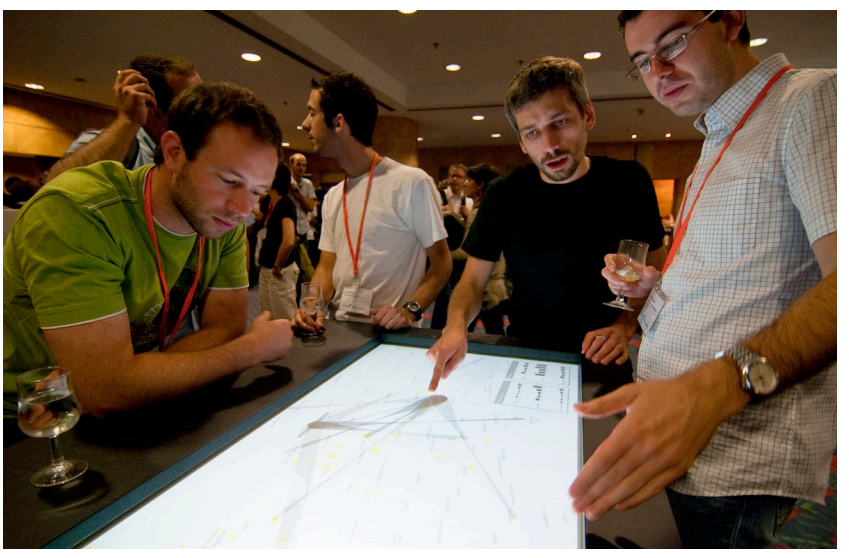

Figure 2: Discussion among conference attendees in a semipublic setting.

In this setting, co-located users, who may or may not be associated with each other, explore the visualization together (Fig. 2). Users can arrive or leave at any time, and have the ability to interact as an individual, or as a 
member of a group with similar interests, goals or attitudes. Cooperative interaction can involve periods of tightly coupled activities by groups with similar but diverging goals, alternated with more loosely coupled individual work. Such collaborative threads can close, split off and merge repeatedly.

The interactive surface of our tabletop has a resolution of 50.8 pixels per inch at full-HD (1080p). With a dimension of $0.96 \times 0.54$ meters multiple persons can gather around to watch and interact with the visualization, simultaneously [28]. The table was designed to be approachable and usable from all sides. At the same time, the table is small enough, that a person can reach all areas on the interactive surface when he stands on one of the wider sides.

\section{Design Approach}

We designed two working prototypes, following an iterative development approach [29], and

demonstrated the first at the EC-TEL conference 2010 in Barcelona, Spain, and the second at the Hypertext conference 2011 in Eindhoven, The Netherlands. The iterative design was chosen in order to refine the spatial network visualizations, and to increase the usability of the interactions. This iterative design, test, redesign, retest cycle allowed us to incorporate feedback from experts and users in all stages. Such an iterative process based on measured use and user comments leads to continuous refinement of the prototype and its features, and helps to make sure that the interactive visualization addresses real needs.

While the iterative methodology itself is well-known, we deliberately went with an open procedure. In all stages, our design decisions were mere offerings to the users.
While based on proven work and prior findings, the whole process was strictly guided by feedback from actual users. Besides validating the interactions and visualizations, it was equally important to learn about the goals and needs of conference attendees.

The demonstration of the prototype at conference locations as well as the collection of feedback from conference visitors lead to us being able to improve the overall experience in direct response. It further allowed us to incorporate the physical and social context of use [14].

\section{Evaluation Methodology}

With both demonstrations, we carried out on-location usability studies with selected conference attendees. We also conducted a preliminary user study on the legibility and distinguishability of visual connection styles. The results from these evaluations helped us to further improve the application to its current state.

We designed the studies as pluralistic usability walkthrough [4], with a semi-structured interview guiding them. Semi-structured interviews give participants the possibility to comment with a degree of freedom [19], but provide more focus than the conversational approach.

As one of the objectives of the visualization is to offer exploration in a collaborative, public setting, we conducted the user study on location, at a place where potential real users are. The advantage of this setting is that it "report[s] on users in their natural environment doing real tasks, demonstrating feasibility and incontext usefulness" [32]. Furthermore, it allowed us to 
observe affective reactions to the prototype, and to ask participants about their experience in a public setting.

We ran the study with one interviewer, and one participant executing the tasks and answering the questions. However, we did not preclude others from watching, or to later join the discussion. This allowed us not only to imitate a live setup at a conference, but also to observe social interactions among the user and bystanders.

We asked the participants to execute selected tasks, and to answer questions concerning the understandability of the visualized information. The five tasks ranged from basic interactions such as navigating to a place, to selecting and exploring institutions, to comparing institutions or countries with each other.

For the two prototypes, these tasks were adapted to the respective data set of conference proceedings, and used comparable affiliations and places (i.e. locations with similar properties and/or visual representations). Slight differences were acceptable, as we were not measuring efficiency.

We observed participants performing the tasks, which allowed us to get a better understanding of the aspects of the problem than if they only have described it verbally [19]. The participants were encouraged to think aloud, while the interviewers were writing down those remarks, as well as their own comments.

Finally, we invited the participants to fill out a post-test questionnaire on their opinions and preferences to determine the perceived usefulness of the visualization. The questionnaire was based on the Useful Satisfaction and Ease-of-Use Questionnaire [21]. We used a 5-point Likert scale with items ranging from "strongly disagree" (1) to "strongly agree" (5). This survey was done privately and anonymously.

The complete sessions took approximately 20-25 minutes each.

\section{First prototype}

The affiliations are shown on a light gray background map. Selected affiliations show their name as label, and their relations as gray connections. A visual connection is shown if authors from two institutions published at least one paper together. There is no indication of the number of collaboratively written papers. Instead, the visual style of the connections varies depending on the overall amount of published papers of both the selected institution and the related institutions (see Fig 3 ).

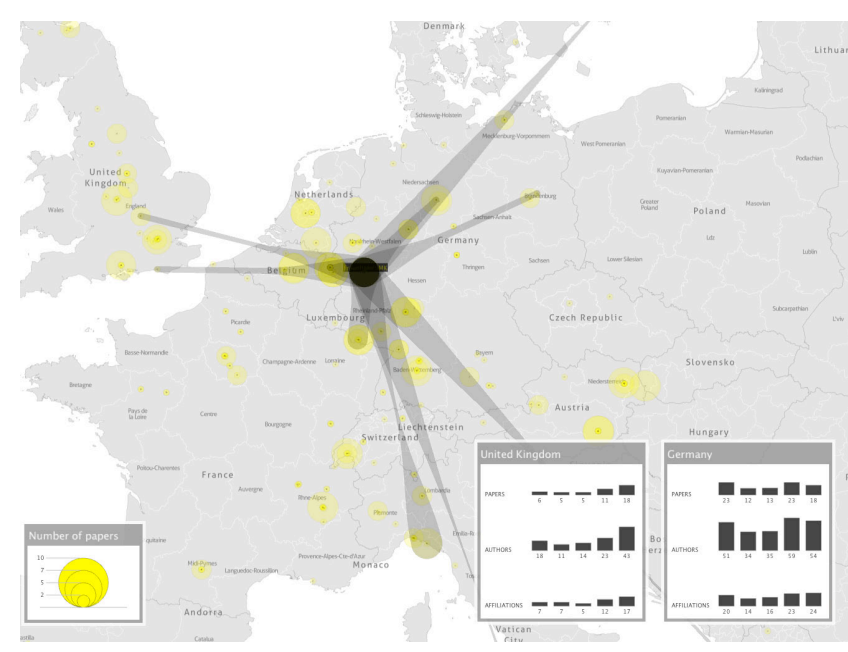

Figure 3: First prototype with one selected institution and its co-authorship connections. 


\section{Evaluation}

We recruited nine male and three female participants, aged 27 to 52 years, from the attendees of the EC-TEL 2010 conference. The participants had normal or corrected-to-normal vision, and all but one were righthanded. All 12 participants had prior experience with touch devices, with eight having further experience with large-scale multi-touch devices.

To assess how the participants related to this specific dataset, we asked for experience and number of publications. With a median of 2 attendances and a mean of 1.5 submitted papers to the EC-TEL conference we assume the participants were engaged adequately to have some personal interest in exploring the visualization.

Seven participants immediately used two finger gestures to zoom and pan the map. While initially two participants tried to double-tap to zoom, two tried using only one finger, and one searched for navigation buttons, they all switched quickly and without cues from the interviewer to the correct interaction mechanisms.

We grouped and unified the feedback and extracted the top three issues, which we discuss in the subsections below. Several improvements are proposed to respond to the detected problems. We identified some further issues, which either only few participants had problems with, or - in one case - were trivial shortcomings: The connection edges were displayed on top of the institution labels, which leads to illegibility if an institution has many connections. This flaw was pointed out by 10 participants, and has been fixed by simply inverting the ordering. Minor issues users commented on included difficulties understanding the switching behavior of the country comparison widget ( 2 participants), the meaning of the year bars (2), and the legend for the circle sizes (1).

\section{QUALITY OF CONNECTIONS UNCLEAR}

The connection between affiliations does not directly represent the number of co-written papers. However, the visual style of the connections varies depending on the overall number of published papers of both the selected institution (with outgoing edges) and the related institutions (incoming) (see Figure 4). We chose this visualization technique with the aim to give an indication which of the connected institutions is the most important one. Evidently, this was not the most adopted interpretation by the users. 10 out of 12 of the participants were under the impression that the connection width visualized the number of co-written papers. Seven participants commented on this, with four suggesting it to be changed with high priority.

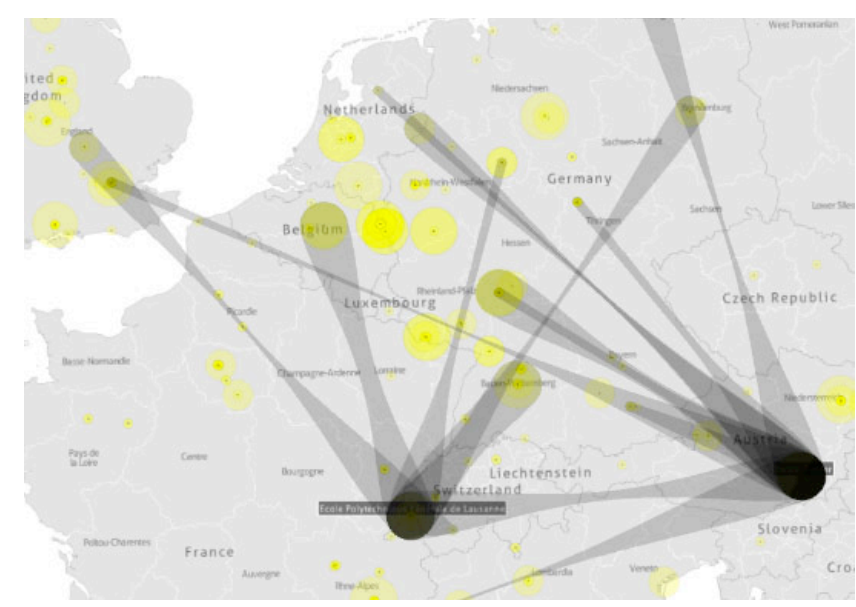

Figure 4: Co-authorship network of two selected institutions. 
Various methods for displaying the strength of these inter-institution relations can be employed; (a) showing the number as text label on the connection, or (b) mapping it onto a graphical variable of the connection representation (e.g. color, or thickness).

INSUFFICIENT GEOGRAPHICAL FEATURES

We chose a map style which displays only few geographical features. The aim of the prototype is to allow exploring and understanding the geo-spatial relations. Thus, the objective of the map is to support general recognition, while being discreet enough to not hinder the display of the data and interface layers.

However, half of the participants had at least some problems in finding places mentioned in the usability walkthrough ${ }^{1}$. This was unexpected, and even though we did not design tasks to examine this issue, specifically, four tasks involved navigating to or identifying a place on the map.

To improve the findability of geographical places, we considered the following possible solutions: (a) Add text field to search for geographical features. This would require some kind of text input, which implicates higher interaction complexity. (b) Display list of selectable countries. This might reintroduce visual clutter, and would only allow filtering on a country level. (c) Display more place names on the map.

\footnotetext{
${ }^{1}$ The interviewer showed or confirmed the correct location, after the participants finished their navigation, to allow them trying
} to answer follow-up question.
ALLOW SINGLE SELECTION OF MULTIPLE AFFILIATIONS ON SAME LOCATION

Tapping a marker selects an affiliation, and shows its name as label, as well as its connections to other affiliations. When asked to select an affiliation, all participants tapped on its marker (with 1 participant double-tapping), and managed to select it correctly. However, users had difficulties selecting a single affiliation if multiple ones are at near-by positions, due to the visual overlapping of markers.

This was due to two reasons: First, insufficient address data provided by the harvested authors, thus not all affiliations could be accurately determined and geopositioned with high precision. Therefore, two institutions from the same city could have been placed at the exact same location (the place's center according to the gazetteer). Second, users also had difficulties when two institutions with separate locations were in close proximity. 10 out of 12 participants could not select "Graz University", where three other affiliations were overlapping. To be able to select one of these by tapping on it with a finger, users first had to zoom in to a level where the markers became distinct.

Solving both issues might be possible in two ways. By (a) changing the layout algorithm in such manner that the markers do not overlap anymore. With many nearby institutions this could lead to a too heavily skewed geo-spatial positioning. Or by (b) clustering the markers, and expanding them, when the user taps on them. 
USEFULNESS AND SATISFACTION

Post-test, we asked the participants to fill out a questionnaire on their satisfaction with the tool, with 11 out of 12 participants responding.

The participants had great fun (median: 5), and were strongly satisfied using Muse (median: 5). Most agreed or strongly agreed (median: 4) to the statement that the visualization helped them to better understand research collaboration.

Overall, the participants strongly found the prototype to be useful (median: 5) and easy to use (median: 5).

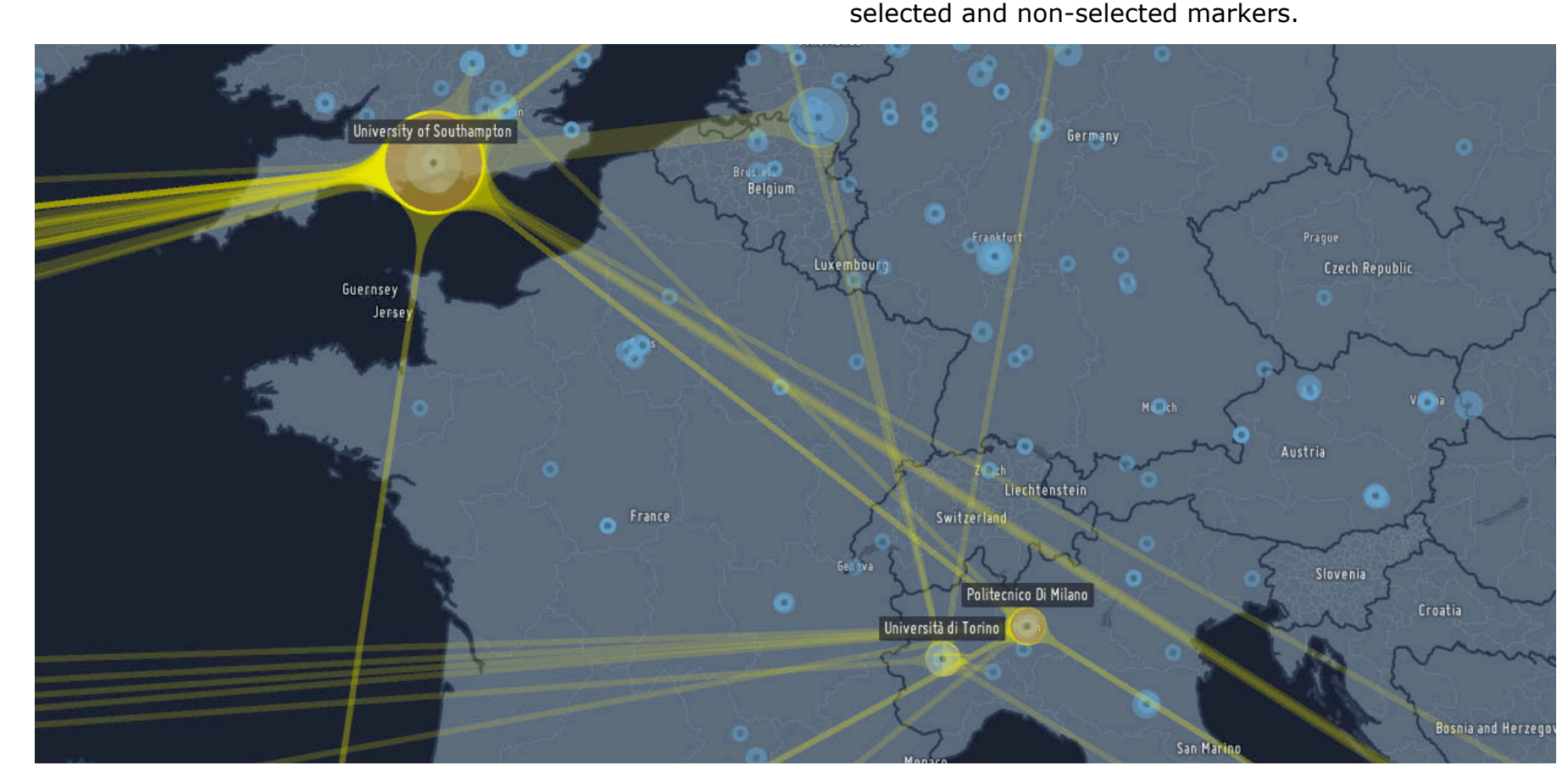

Fig. 5: Second prototype with selected and non selected institutions.

\section{Second prototype}

In the next iteration, we tackled the identified issues, reviewed papers reporting on related studies, and tried to overcome the problems by implementing one of the solutions proposed above.

In order to allow correct data interpretation, and the comparison of different markers, the visual encoding is based on a power law suitable for symbol size

discrimination [20]. We based the size of the text labels on findings of Ashdown et al [1], and selected a font size of $14 \mathrm{pt}$ for a display resolution of 50ppi.

Furthermore, we added a visual distinction between selected and non-selected markers. 
VISUALIZE QUALITY OF CONNECTIONS

Relations between places are visualized by connecting lines between the two markers (Fig. 5). In our case study, a visual connection is shown if authors from two institutions published at least one paper together. The thickness of the connections varies depending on the total number of co-published papers of the selected institution with the related institutions. The results of a user study with an earlier prototype lead us to adapt the visualization to map the strength of a connection.

The visual style was chosen for good legibility, while the smooth junctions between the circle marker and the connection aim for an aesthetically pleasing look of connectedness [31]. It was also chosen to make it easier discernible whether a connection merely passes under a marker, i.e. points to another affiliation in the same direction. Through the enlargement of the line in proximity of a marker, it aims to make it clearly visible to which marker the line belongs to. The lines connect two institutions transparently, in order to not obstruct the underlying map or markers
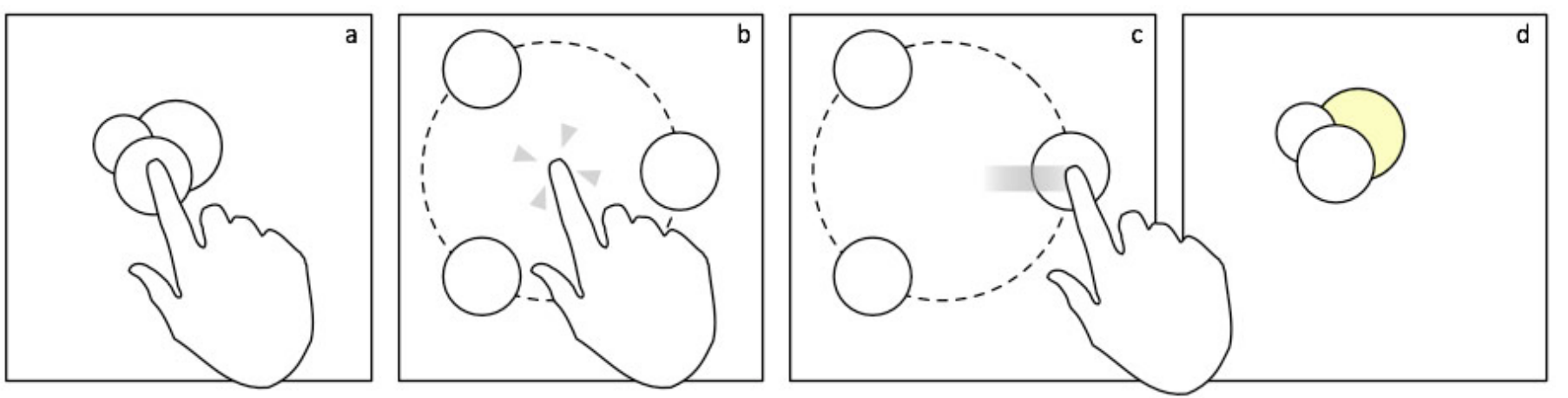

IMPROVE MAP STYLE AND ADD GEOGRAPHIC PLACES We used a map showing more labels of geographical places. We adapted the map design to fit to the rest of our visualization according to color schema and typeface. It has to be verified whether the least demanding technical and cognitive solution of using a more detailed map style will be sufficient to help users find the places and institutions they are interested in.

ENABLE SELECTION OF SINGLE MARKER IN A CLUSTER Places are all shown at their original geo-location, which results in overlapping markers for institution in close proximity. In contrast to non-geographic layout strategies, where a positioning algorithm may prevent collisions, we opted for a visualization technique in which the visual distribution shows spatial patterns. However, precise selection of small or overlapping markers is difficult due to the fat finger problem [34].

This was also evident in the behavior of the participants in our user study of our first prototype. Thus, we designed Exploding Menu, a mechanism to ease the selection of near-by institutions.

Figure 6: Selecting an institution from a group of near-by marker 


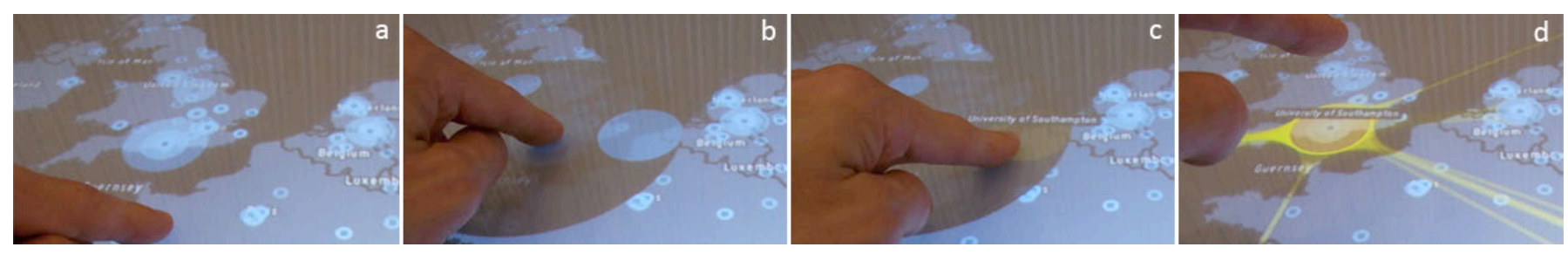

Figure 7: Selecting an institution from a group of near-by markers in the second prototype

In the beginning, multiple overlapping markers are shown at one location (Fig. 6a). When a user touches the marker cluster, a radial menu appears with all the markers evenly laid out on a concentric ring (Fig. 6b). The user slides (or taps with a second finger) onto the markers, which will be highlighted (Fig. 6c). When the user releases his finger atop one menu item the marker gets selected, and is shown at its original position (Fig. $6 d)$. To select another marker of the same cluster, the interaction pattern has to be repeated. The same mechanism can be used to deselect institutions.

In our prototype, all markers represent research institutions with their number of publications mapped to the size of the marker. In Figure 7a, multiple markers with various sizes are shown at the south of Great Britain. The user taps on the cluster, and the radial menu is displayed with a black transparent circle, darkening the background map and other markers (Fig. $7 b)$. Note that the marker proxies in the menu are shown in their original sizes. When the user slides over of the proxy markers, the name of the institution is displayed (Fig. 7c) in order to support selecting the correct affiliation. After the user selects one marker the radial menu disappears, and the corresponding institution is shown with all its connections (Fig. 7d).

\section{Social Interactions}

The tabletop visualization induced not only humancomputer, but also human-human interaction. We observed a variety of ways in which attendees got in touch with each other. Users were:

- $\quad$ Starting discussions. When users were exploring their personal network, observers commented on connections to their own institution, or on shared collaborators. Often, this resulted in lively conversations.

- Exploiting the visualization for story-telling. For example, one user was narrating the history of his institution over the last 15 years and its contribution to his field to fellow researchers, while selecting affiliations and pointing to connections.

- Engaging other users. When one or more persons were actively interacting with the table, passers-by were more inclined to stay for a bit, watched the visualization and the interactions, and eventually tried it out themselves.

\section{Evaluation}

At the Hypertext 2011 conference, we conducted a similar usability walk-through as for the first prototype, and investigated if the changes improved the usability of the visualization. 
The evaluation study involved nine participants, two females, and seven males, aged 23 to 44 years. The participants had great fun (median: 5), and were satisfied using Muse (median: 4). Most agreed or strongly agreed (median: 4) to the statement that the visualization was useful in reflecting on the Hypertext community, and that is was very useful (median: 5) in understanding the geospatial spread of the research network. Overall, the participants strongly considered the prototype easy to use (median: 4 ).

\section{Conclusion and Outlook}

We presented two working prototypes for exploring geospatial networks. We designed these as walk-upand-use systems with comprehensible geo-

visualization, so that interested stakeholders can use it without much effort. It was designed for a large multitouch tabletop, in order to invite users to participate and engage in discussions at a semi-public location.

In our case study, the application visualizes coauthorship data of conference publications. The geographic distribution of the institutions, as well as the visualization of the number of publications has been found to be easily understandable. Through interactive filtering, the users were able to explore the relations between their affiliations and other institutions, and could gather insights into the collaboration network in their domain.

The results of our usability studies, and the feedback gathered from the questionnaire demonstrate that this is a promising approach to exploring geospatial relationships in scientific networks.
In designing the system, we learnt valuable lessons, which we summarize below.

\section{Rapid Adaption of Map Styles}

We did not expect the problems participants had with orienting themselves on an interactive world map. One design challenge is to balance the style and detail levels of a geographic map for geovisualization, providing enough details to enable users to recognize places, and not so many details that they interfere with the information overlaid over the map. Having the ability to adapt the map style rapidly to our dataset was very helpful.

\section{Visual Style of Weighted Connections}

The design of the connections between institutions is based on a preliminary user study. As we are not aware of any controlled user study on the legibility of weighted connections, we emphasize the need for further research in this field.

Acceptance of Multitouch Interaction

Compared to other recent studies ([27], [10], [37]), the participants of our studies had more previous experience with multitouch devices. This was reflected in their behavior, as few were having problems with the map navigation. For a user group of tech-savvy persons, we thus feel it is by now quite safe to deploy multitouch interaction in large-screen visualizations.

Radial Menu for Dense Geospatial Data

Our solution for densely positioned markers on a multitouch tabletop used a technique similar to existing ones. However, applying a solution for imprecise finger selection in the context of geospatial data was novel. 


\section{Design Process}

The iterative design with demonstrations at different conferences was helpful in gathering continuous feedback, but also helped to create a more flexible visualization for different data sets (e.g. finding solutions for dense geo-data). In this way, our pragmatic, iterative process was very effective and we thus feel confident to encourage others to quickly demonstrate early prototypes in the target setting, and constantly refine the techniques used.

We applied several existing techniques, ranging from multitouch interactions to network visualization, and adapted them for the context of geo-located coauthorship data visualization on a tabletop. While many of the techniques used are well-established, we see their aesthetic and usable composition in a conference setting as a successful design case study.

\section{References}

[1] Ashdown, M., Tuddenham, P. and Robinson, P. 2010. High-Resolution Interactive Display. In: MüllerTonfelde, C. (ed.) Tabletops - Horizontal Interactive Displays, HCIS, Part 1, 71-100, Springer

[2] Batty, M. 2002. The Geography of Scientific Citation. Environment and Planning: A 35:761-765

[3] Beauchesne, O. 2011. Map of scientific collaboration between researchers.

http://olihb.com/2011/01/23/map-of-scientificcollaboration-between-researchers/

[4] Bias, R.G. The pluralistic usability walkthrough: Coordinated Empathies. 1994. In Usability Inspection
Outlook

We used the radial exploding menu to solve overlapping map markers in a different visualization (i.e. enabling the precise selection of bus stations in a public transit application). In a next step, we are going to evaluate how well this technique can be transferred to other domains, and how well it performs compared to alternative selection methods.

To guide our selection of the visual mapping indicating the strength of a connection between institutions, we conducted a preliminary user study on various display styles. Currently, we are planning an extended and more general user study on weighted edge visualization.

\section{Acknowledgements}

We like to thank all participants of our studies. We also like to thank the anonymous reviewers for their valuable feedback, and Bram Vandeputte for providing an interactive tabletop.

Methods, J. Nielsen and R. Mack (eds), p63-76, Wiley and Sons

[5] Börner, K. 2010. Atlas of Science : visualizing what we know. MIT Press, Cambridge

[6] Chen, C. 2004. Information Visuzalization. 2nd ed. Springer, London

[7] Chen, C. 1999. Trailblazing the Literature of Hypertext: Author Co-Citation Analysis (1989-1998). HT 1999

[8] citeulike. http://www.citeulike.org/

[9] Cyberinfrastructure for Network Science Center. 2009. 
http://scimaps.org/maps/map/research_collaborati_11

[10] Echtler, F., Huber, M. and Klinker, G. 2008. Shadow Tracking on Multi-Touch Tables. In Proc of AVI 2008. ACM Press, doi:1-978-60558-141-5

[11] Elmqvist, N., Vande Moere, A., Jetter, H.-C. Cernea, D., Reiterer, H. Jankun-Kelly, T. J. Fluid Interaction for Information Visualization. Information Visualization Journal (IVS), Special Issue on Information Visualization: State of the Field and New Research Directions, 10(4):327-340, October 2011

[12] Freeman, L. 2000. Visualizing Social Networks. Journal of Social Structure, 1

[13] Glänzel, W., Schubert, A. 2004. Analyzing Scientific Networks through Co-authorship. In Handbook of Quantitative Science and Technology Research, H.F. Moed et al. (eds.), 257-276

[14] Gulliksen, J., Göransson, B., Boivie, I., Blomkvist, S., Persson, J., Cajander, A. 2003. Key principles for user-centred systems design. Behaviour and Information Technology 22 (6), 397-410

[15] Haklay, M. and Weber, P. 2008. OpenStreetMap: User-Generated Street Maps. In IEEE Pervasive Computing, vol. 7, no. 4, pp. 12-18, Oct.-Dec. 2008, doi:10.1109/MPRV.2008.80

[16] Henry, N., Goodell, H., Elmqvist, N., Fekete, J.-D. 2007. 20 Years of Four HCI Conferences: A Visual Exploration. International Journal of Human-Computer Interaction, 23:3, 239-285

[17] Hotho, A., Jschke, R., Schmitz, C. and Stumme, G. 2006. BibSonomy: A Social Bookmark and Publication Sharing System. In Proc. of the Conceptual Structures Tool Interoperability Workshop at the 14th Int. Conf. on Conceptual Structures

[18] Katz, J. S. 1994. Geographical Proximity and Scientific Collaboration. In Scientometrics 31 (1), 31 43
[19] Lazar, J, Feng, J. and Hochheiser, H. 2010. Research Methods in Human Computer Interaction. Wiley and Sons

[20] Li, J., Martens, J.-B., van Wijk, J.J. 2010. A model of symbol size discrimination in scatterplots. CHI 2010 : 2553-2562

[21] Lund, A. M. 2001. Measuring usability with the USE questionnaire. Usability Interface: Usability SIG Newsletter, October.

[22] Luukkonen, T., Persson, O. and Sivertsen, G. 1992 Understanding patterns of international scientific

collaboration. Science Technology Human Values, 17: 101-126

[23] MacEachren, A., Gahegan, M., Pike, W., Brewer, I. Cai, G., Lengerich, E. 2004. Geovisualization for Knowledge Construction and Decision Support. IEEE CG\&A 2004; 1: 13-17

[24] MapBox. http://mapbox.com/

[25] Melin, G., 2000. Pragmatism and self-organization: research collaboration on the individual level. Research Policy 29, 31-40

[26] Mendeley. http://www.mendeley.com/

[27] Moscovich, T. and Hughes, J. F. 2008. Indirect Mappings of Multi-touch Input Using One and Two Hands. In Proc. of CHI 2008. ACM Press, doi:1-59593178-3/07/0004

[28] Nagel, T., Pschetz, L., Stefaner, M., Halkia, M. Müller, B. 2009. mæve - An Interactive Tabletop Installation for Exploring Background Information in Exhibitions. In Proc. HCII 2009. doi:10.1007/978-3642-02580-8_53

[29] Nielsen, J. 1993. Iterative User Interface Design. IEEE Computer vol.26 no.11 pp 32-41

[30] Ochoa, X., Méndez, G., Duval, E. 2009. Who We Are: Analysis of 10 Years of the ED-MEDIA Conference. In Proc. of World Conference on Educational Multimedia, Hypermedia and Telecommunications, 189200 
[31] Palmer, S. E., and Rock, I. 1994. Rethinking perceptual organization: The role of uniform

connectedness. Psychonomic Bulletin and Review, 1 29-55

[32] Plaisant, C. 2004. The Challenges of Information Visulization Evaluation. In IEEE Proc. of AVI 2004

[33] Ponds, R., Van Oort, F. G., Frenken, K. 2007. The geographical and institutional proximity of research collaboration. Papers in Regional Science, 86(3), 423443

[34] Remy, C., Weiss, M., Ziefle, M., Borchers, J. 2010.

A Pattern Language for Interaction Tabletops in

Collaborative Workspaces. EuroPLoP 2010
[35] Shen, Y. 2006. Scholarly communication in scientific research practice - a study of computer sciences faculty. Libri 56: 239-251

[36] Walsh, J. P., S. Kucker and N. G. Maloney 2000.

Connecting minds: Computer-mediated communication and scientific work. Journal of the American Society for Information Science 51(14): 1295-1305

[37] Wang, F., Cao, X., Ren, X. and Irani, P. 2009. Detecting and Leveraging Finger Orientation for

Interaction with Direct-Touch Surfaces. In Proc. of UIST 2009, ACM Press, doi:978-1-60558-745-5/09/10

[38] Web-Harvest, http://web-harvest.sourceforge.net/ [39] White, S.

http://catalysoft.com/articles/StrikeAMatch.html 